\title{
Endoscopic Mucosal Resection
}

National Cancer Institute

\section{Source}

National Cancer Institute. Endoscopic Mucosal Resection. NCI Thesaurus. Code C103242.

An endoscopic procedure that visualizes a part of the gastrointestinal tract to first identify and demarcate superficial mucosal lesions, then to guide a submucosal injection to the site to lift the lesion and finally to aid in the removal of the lesion. 